\title{
Implementing Models of Geriatric Care-Behind the Scenes
}

\author{
Joshua Chodosh, MD, MSHS, ** and Michael Weiner, MD, MPH*s
}

Innovative geriatric clinical programs have proliferated in the 21st century, and many have been highlighted in the Journal of the American Geriatrics Society (JAGS). The Affordable Care Act has supported the accelerated innovation of publicized and unpublicized program development, adaptation, and implementation. Many JAGS articles report work conducted in programs with significant improvements in quality; high satisfaction for patients and providers; and for some, reductions in costs. Despite considerable detail, enabling implementers to attempt to adopt reported programs or adapt them to local environments, much less is typically conveyed about the subtleties of the implementation process that led to a successful outcome. Moreover, where we have been given a window into successful initiatives, far less is known about those that failed and even less about why some succeeded but others failed. With a focus on our shared needs as a geriatrics community, to foster the exchange of more-comprehensive models of successful and failed implementation, we propose publications that address implementation itself - a second layer of reporting about the "hidden" elements that may have been decisive factors in taking an efficacious test, treatment, or model and putting it into real-world practice. We propose a new platform for sharing a broader range of healthcare quality improvement initiatives-successes and failures. We include several salient characteristics that could be measured and described in support of dynamic, sustainable, evidence-based implementation of geriatrics programs. J Am Geriatr Soc 66:364-366, 2018.

From the "Division of Geriatric Medicine and Palliative Care, School of Medicine, New York University, New York, New York; ${ }^{\dagger}$ Department of Medicine, Veterans Affairs New York Harbor Healthcare System Manhattan Campus, New York, New York; ${ }^{\star}$ Department of Medicine, School of Medicine, Indiana University, Indianapolis, Indiana; and ${ }^{\S}$ Health Services Research and Development Service, CIN 13-416, Center for Health Information and Communication, Richard L. Roudebush Veterans Affairs Medical Center, Veterans Health Administration, U.S. Department of Veterans Affairs, Indianapolis, Indiana.

Address correspondence to Joshua Chodosh, Division of Geriatric Medicine and Palliative Care, School of Medicine, New York University, Department of Medicine, Veterans Affairs New York Harbor Healthcare System Manhattan Campus, 550 First Avenue, BCD616, New York, NY 10016. E-mail: joshua.chodosh@nyumc.org

DOI: $10.1111 /$ jgs. 15183
Key words: geriatrics; implementation science; models of care

$\mathrm{H}$ ealth care in the United States has seen many changes in the 21st century, accelerated by the Affordable Care Act and a genuine interest in improving the quality of healthcare for all Americans. Many of these changes, some initiated before 2000, have occurred in settings focused on older-age care and have included innovative programs with well-known names such as Program of All-inclusive Care for the Elderly, ${ }^{1}$ Geriatric Resources for Assessment and Care of Elders, ${ }^{2}$ Improving Mood-Promoting Access to Collaborative Treatment, ${ }^{3}$ Care Transitions, ${ }^{4,5}$ and Interventions to Reduce Acute Care Transfers. ${ }^{6,7}$ Although adaptations of these and other programs have proliferated, most instances of implementation of these programs are unlikely to have received notice beyond their local sites and surrounding communities. The Journal of the American Geriatrics Society (JAGS) has born witness to a cross-section of this movement with several related publications, including eight Models of Care articles in 2016 alone.

These publications represent the successful efforts of healthcare practitioners, administrators, and patients who participated in the implementation and evaluation of innovative ideas. We know from our own experiences and those of our colleagues that JAGS and other journals have not published numerous reports of additional initiatives, because the quality of evaluation or its documentation was insufficient to merit publication; the implementers, although successful, chose not to submit their work for publication; or the implementation did not reach a level of achievement deemed worthy of documentation. Other initiatives may have failed to achieve goals that their originators established. Whether successful or not, only a fraction of these efforts of our healthcare colleagues who toil to innovate and improve care are likely to be widely known.

As clinical geriatrics programs increasingly move evidence into everyday practice, geriatricians and their healthcare teams and partners face many of the implementationrelated challenges of other disciplines. Geriatrics providers 
are challenged in busy clinical environments, striving to integrate new research findings into the care that they provide in clinics, hospitals, extended-care facilities, communities, and patients' homes. At the institutional level, many geriatricians have assumed leadership roles as a reflection of increasing awareness of the need to address systematically the healthcare problems that cannot be solved without large-scale structural solutions. ${ }^{8,9}$

Whereas local context will dictate the need for a nuanced approach to implementation, many of the principles necessary for a successful strategy are broadly applicable, yet too little has been done to articulate and disseminate these principles beyond the more academic contributions of implementation science. ${ }^{10,11}$ Moreover, without more public sharing of successes, failures, the myriad of challenges, and the strategies deployed to meet those challenges, random activities, duplicative approaches less likely to be successful, and related inefficiencies will limit the success of well-intentioned implementers.

The field of implementation science has provided considerable guidance for considering, planning, executing, and sustaining an intervention. The well-known Consolidated Framework for Implementation Research ${ }^{12-14}$ comprises five domains: intervention, inner setting, outer setting, individuals involved, and implementation process (Table 1). This framework supports an important approach to implementation for design and evaluation, but learning more about a framework like this, and applying it, can be challenging; a full delineation of this evaluative framework is beyond the scope and intent of many descriptive publications about models of care, and many implementers lack the resources needed for a detailed evaluation that would address the framework's domains with any substantive depth.

To help implementers make progress in understanding and adapting useful models, single-site clinical programs are often described in JAGS in the Models of Care section. These are often highly successful, as significant improvements in quality; high satisfaction for patients and providers; and for some, reductions in costs indicate. Protocols may often be specific enough that motivated clinicians or administrators can replicate, often with local adaptations, these programs at their home institutions, with varying degrees of success. What is often replicated is an interdisciplinary model representing the skillsets of nurses, social workers, pharmacists, rehabilitative therapists, physicians, and other health professionals, such as psychologists, coaches, navigators, educators, "case managers," and health technicians. Community health workers are an increasingly important and recognized resource as we gain precision and clarity about essential clinical processes and the roles best suited to performing those processes. ${ }^{715-17}$ Overall, these interdisciplinary approaches reflect the complexities of need of our oldest, frailest patients. Robust protocols and supporting infrastructure, enhanced by health information technology, informatics, engineering, and human factors, reduce or accommodate certain complexities, and the ensuing organizational programmatic structure can increase the likelihood of success. Although these ingredients are essential for successful programs, they do not necessarily address the essential process of implementation, the human skills needed for achieving successful and sustainable programs, and the challenges that are ever-present in our complex health systems. In other words, what really happened and what conclusions can be drawn from these experiences may be left unsaid.

With a focus on our shared needs as a geriatrics community, to exchange models of successful and failed implementation, we propose publications that address the implementation itself-a second layer of reporting about those "hidden" elements that may have been decisive factors in taking an efficacious test, treatment, or model and putting it into real-world practice. How did the process begin and with what construct or framework? How did it proceed and advance? What data were important for implementing, disseminating, and sustaining the program? In pursuing an agenda for healthcare innovation that relies on geriatric clinical knowledge, how were interpersonal sensitivities, and an acute awareness of formal and informal institutional structures that drive and impede otherwise sensible solutions to healthcare delivery problems,

\begin{tabular}{|c|c|}
\hline Constructs & Definition \\
\hline $\begin{array}{l}\text { Intervention } \\
\text { characteristics }\end{array}$ & $\begin{array}{l}\text { Core (immutable) components; adapted } \\
\text { components }\end{array}$ \\
\hline Outer setting & $\begin{array}{l}\text { Economic, political, social context surrounding } \\
\text { an organization }\end{array}$ \\
\hline Inner setting & $\begin{array}{l}\text { Structure, networks - communications, culture, } \\
\text { mood, readiness }\end{array}$ \\
\hline $\begin{array}{l}\text { Participant } \\
\text { characteristics }\end{array}$ & Targeted users, others who are affected \\
\hline $\begin{array}{l}\text { Implementation } \\
\text { process }\end{array}$ & $\begin{array}{l}\text { Planned formal, informal activities, intended and } \\
\text { unintended actions }\end{array}$ \\
\hline
\end{tabular}

Table 2. Proposed Additional Reported Characteristics of Models of Care Implementation

\begin{tabular}{ll}
\hline \multicolumn{1}{c}{ Constructs } & \multicolumn{1}{c}{ Definition } \\
\hline Initiating conditions & $\begin{array}{l}\text { Context, circumstances that made the } \\
\text { opportunity possible }\end{array}$ \\
$\begin{array}{l}\text { Skills, experiences of } \\
\text { implementers }\end{array}$ & $\begin{array}{l}\text { Description of all contributing individuals } \\
\text { and their professional (formal) and } \\
\text { personal (informal) skills and } \\
\text { implementation experience }\end{array}$ \\
Interpersonal challenges & $\begin{array}{l}\text { Challenges internal to team functioning } \\
\text { and external to implementation team and } \\
\text { how those challenges were met }\end{array}$ \\
Unique facilitators, barriers & $\begin{array}{l}\text { Unique circumstances - structures or } \\
\text { events-that served as a facilitator or } \\
\text { barrier and how that was leveraged or } \\
\text { addressed }\end{array}$ \\
Surprising conditions, \\
events
\end{tabular}$\quad \begin{aligned} & \text { What occurred or was discovered that } \\
& \text { was not anticipated; what role or } \\
& \text { influence, if any, that condition or event } \\
& \text { played in the implementation } \\
& \text { What conditions or events raise concerns } \\
& \text { about a program's survival; how these } \\
& \text { are, or are not, being addressed } \\
& \text { What other resources or activities are } \\
& \text { requirements for, }\end{aligned}$


leveraged effectively? Where did the process fail? What unique opportunities shaped or forced an evolution that may not be present in other institutions? More than the model itself, the model's supports, requirements, surroundings, relationships, challenges, and opportunities would be the focus of these implementation publications (Table 2). This meta-process may be as important as documentation of the implementation process. We challenge local, regional, national, and international leaders who have implemented or disseminated innovative models of care to share, describe, and discuss their implementation processes, measurements of those processes, and dissemination of findings that speak to the challenges that identify not only the successes but also the failures, to prepare future implementers, and to expand this burgeoning field of creative, adaptive, dynamic, sustainable, evidence-based geriatrics implementation.

\section{ACKNOWLEDGMENTS}

We give thanks to the hard working geriatrics professional community that has provided countless hours in addition to patient care to develop and implement new approaches to care to improve the lives of patients and others like them.

Conflicts of Interest: None.

Author Contributions: Dr. Chodosh had full access to all literature used in this study and takes responsibility for the integrity and accuracy of its representation. Chodosh and Weiner: study concept and design, drafting of manuscript, literature interpretation, critical revision of manuscript.

Sponsor's Role: Not applicable.

\section{REFERENCES}

1. Lee W, Eng C, Fox $N$ et al. PACE: A model for integrated care of frail older patients. Program of all-inclusive care for the elderly. Geriatrics 1998;53:62-73.

2. Counsell SR, Callahan CM, Buttar AB et al. Geriatric Resources for Assessment and Care of Elders (GRACE): A new model of primary care for low-income seniors. J Am Geriatr Soc 2006;54:1136-1141.
3. Unützer J, Katon W, Callahan CM et al. Collaborative care management of late-life depression in the primary care setting: A randomized controlled trial. JAMA 2002;288:2836-2845.

4. Coleman EA, Smith JD, Frank JC et al. Preparing patients and caregivers to participate in care delivered across settings: The care transitions intervention. J Am Geriatr Soc 2004;52:1817-1825.

5. Coleman EA, Roman SP, Hall KA et al. Enhancing the care transitions intervention protocol to better address the needs of family caregivers. J Healthc Qual 2015;37:2-11.

6. Ouslander JG, Bonner A, Herndon L et al. The INTERACT quality improvement program: An overview for medical directors and primary care clinicians in long-term care. J Am Med Dir Assoc 2014;15:162-170.

7. Walsh EG, Wiener JM, Haber S et al. Potentially avoidable hospitalizations of dually eligible Medicare and Medicaid beneficiaries from nursing facility and Home- and Community-Based Services waiver programs. J Am Geriatr Soc 2012;60:821-829.

8. Davidson GH, Austin E, Thornblade L et al. Improving transitions of care across the spectrum of healthcare delivery: A multidisciplinary approach to understanding variability in outcomes across hospitals and skilled nursing facilities. Am J Surg 2017;213:910-914.

9. Kennedy MP. Implementation of quality improvement methodology and the medical profession. J Qual Clin Pract 1998;18:143-150.

10. Ritchie C, Andersen R, Eng J et al. Implementation of an interdisciplinary, team-based complex care support health care model at an academic medical center: Impact on health care utilization and quality of life. PLoS ONE 2016;11:e0148096.

11. Tan ZS, Damron-Rodriguez J, Cadogan $M$ et al. Team-Based interprofessional competency training for dementia screening and management. J Am Geriatr Soc 2017;65:207-211.

12. Damschroder LJ, Aron DC, Keith RE et al. Fostering implementation of health services research findings into practice: A consolidated framework for advancing implementation science. Implement Sci 2009;4:50.

13. Keith RE, Crosson JC, O'Malley AS et al. Using the Consolidated Framework for Implementation Research (CFIR) to produce actionable findings: A rapid-cycle evaluation approach to improving implementation. Implement Sci 2017;12:15.

14. Kirk MA, Kelley C, Yankey $\mathrm{N}$ et al. A systematic review of the use of the Consolidated Framework for Implementation Research. Implement Sci 2016;11:72.

15. Islam N, Nadkarni SK, Zahn D et al. Integrating community health workers within patient protection and Affordable Care Act implementation. J Public Health Manag Pract 2015;21:42-50.

16. Kane EP, Collinsworth AW, Schmidt KL et al. Improving diabetes care and outcomes with community health workers. Fam Pract 2016;33:523-528.

17. Katigbak C, Van Devanter N, Islam N et al. Partners in health: A conceptual framework for the role of community health workers in facilitating patients' adoption of healthy behaviors. Am J Public Health 2015;105: $872-880$. 